\title{
Compositional Derivation of Symmetries for Constraint Satisfaction
}

\author{
Pascal Van Hentenryck ${ }^{1}$, Pierre Flener $^{2}$, \\ Justin Pearson ${ }^{2}$, and Magnus Ågren ${ }^{2}$ \\ 1 Department of Computer Science \\ Brown University, Box 1910, Providence, RI 02912, USA \\ pvh@cs.brown.edu \\ 2 Department of Information Technology \\ Uppsala University, Box 337, 75105 Uppsala, Sweden \\ \{pierref, justin, agren\}@it.uu.se
}

\begin{abstract}
This paper reconsiders the problems of discovering symmetries in constraint satisfaction problems (CSPs). It proposes a compositional approach which derives symmetries of the applications from primitive constraints. Its key insight is the recognition of the special role of global constraints in symmetry detection. Once the symmetries of global constraints are available, it often becomes much easier to derive symmetries compositionally and efficiently. The paper demonstrates the potential of this approach by studying several classes of value and variable symmetries and applying the resulting techniques to two non-trivial applications. The paper also discusses the potential of reformulations and high-level modelling abstractions to strengthen symmetry discovery.
\end{abstract}




\title{
Compositional Derivation of Symmetries for Constraint Satisfaction
}

\author{
Content Area: Constraint Satisfaction
}

\begin{abstract}
This paper reconsiders the problems of discovering symmetries in constraint satisfaction problems (CSPs). It proposes a compositional approach which derives symmetries of the applications from primitive constraints. Its key insight is the recognition of the special role of global constraints in symmetry detection. Once the symmetries of global constraints are available, it often becomes much easier to derive symmetries compositionally and efficiently. The paper demonstrates the potential of this approach by studying several classes of value and variable symmetries and applying the resulting techniques to two non-trivial applications. The paper also discusses the potential of reformulations and high-level modeling abstractions to strengthen symmetry discovery.
\end{abstract}

\section{Introduction}

Many applications in constraint satisfaction exhibit natural symmetries which may significantly increase the difficulty of solving. It is thus not surprising that increased attention has been devoted to symmetry breaking in the last decade.

Recent research has mostly focused on breaking symmetries, including general symmetry-breaking schemes (e.g., SBDS (Backofen \& Will 1999; Gent \& Smith 2000) and SBDD (Fahle, Schamberger, \& Sellmann 2001; Focacci \& Milano 2001)), their efficient implementations (e.g., (Puget 2003)), and their specialisations for specific applications (e.g., (Barnier \& Brisset 2002; Puget 2002)). There has also been a tendency to abstract some of the techniques from particular applications to classes of CSPs (Van Hentenryck et al. 2003) or models (Flener et al. 2002). However, this line of research assumes that symmetries are given and ignores the tedious and error-prone task of discovering them.

The detection of symmetries is a research avenue pioneered by (Freuder 1991) and subsequently investigated by many others. Freuder introduced various forms of value interchangeability and his goal was to discover symmetries and preprocess CSPs to remove their symmetries. Unfortunately, it is not tractable to discover many, apparently simple, classes of symmetries in CSPs arising in practical applications.

This research reconsiders the problem of discovering symmetries from a fundamentally novel angle. Its key in-

Copyright (c) 2004, American Association for Artificial Intelligence (www.aaai.org). All rights reserved. sight is to recognize that global (optimization) constraints (Beldiceanu \& Contejean 1994; Régin 1994; Focacci, Lodi, \& Milano 2002) offer significant benefits for deriving symmetries compositionally and efficiently. Global constraints are a fundamental aspect of constraint programming: They capture common combinatorial substructures of practical applications and exploit the substructure semantics to obtain more effective filtering algorithms, linear relaxations, and cooperation schemes between solvers. The main contribution of this research is to show that, once the symmetries of global constraints are specified, it becomes much simpler to derive the symmetries of an application. This research can also be seen as shifting the burden of discovering symmetries from users to solver designers who are experts in the underlying combinatorics.

The purpose of this paper is to demonstrate the potential of this research direction. The paper makes the following technical contributions:

1. It considers various classes of symmetries and shows how to derive symmetries compositionally and efficiently, starting from global constraints. They include value and variable symmetries, and symmetries in matrix models.

2. It shows how to apply these results to derive the symmetries of two non-trivial applications: scene allocation and progressive party.

3. It shows how various problem reformulations can improve the accuracy of the derivations and suggests a variety of modeling practices to improve symmetry detection.

These technical results should be viewed as a first (small) step towards a comprehensive automated tool for discovering symmetries. What is particularly interesting however is their ability to handle non-trivial applications already, as well as the various research directions they suggest for modeling languages and reformulation tools.

The rest of the paper is organized as follows. After some preliminaries, the paper shows how constraint and function symmetries can be composed for various forms of interchangeability. The techniques are then illustrated on two applications: scene allocation and the progressive party problem. The next section discusses how problem reformulations improve symmetry detection. Finally, symmetries in matrix models are presented and illustrated. 


\section{Preliminaries}

This section defines the main concepts used in this paper. The definitions are borrowed from (Van Hentenryck et al. 2003), which uses them for different purposes. The basic idea is to abstract the set of constraints by a Boolean function which holds if all the constraints are satisfied. Solutions are also represented as functions (assignments), namely from variables to the set of values.

Definition 1. A $C S P$ is a triple $\langle V, D, C\rangle$, where $V$ denotes the set of variables, $D$ denotes the set of possible values for these variables, and $C:(V \rightarrow D) \rightarrow B o o l$ is a constraint that specifies which assignments of values to the variables are solutions. A solution to a $\operatorname{CSP} \mathcal{P}=\langle V, D, C\rangle$ is a function $\sigma: V \rightarrow D$ such that $C(\sigma)=$ true. The set of solutions to a $\operatorname{CSP} \mathcal{P}$ is denoted by $\operatorname{Sol}(\mathcal{P})$.

Many practical problems involve the optimization of objective functions and much research in recent years has focused on applying filtering algorithms to prune the resulting "optimization” constraints (e.g., (Régin 1999; Focacci, Lodi, \& Milano 2002)). In general, in existing languages and systems, these optimization constraints are expressed using auxiliary variables. However, it is more elegant from a modeling standpoint, and more effective when deriving symmetries, to capture these functions directly.

Definition 2. A global function over variables $V$ and values $D$ is a function $f:(V \rightarrow D) \rightarrow \mathcal{N}$.

A constraint optimization problem (COP) consists of minimizing an objective function subject to a set of constraints.

Definition 3. A $C O P$ is a quadruple $\mathcal{O}=\langle V, D, C, f\rangle$, where $\mathcal{P}=\langle V, D, C\rangle$ is a CSP and $f$ is a global function over $V$ and $D$. The optimal value $f^{*}$ of $\mathcal{O}$ is the minimal value of $f$ taken by any solution to $\mathcal{P}$, i.e.,

$$
f^{*}=\min _{\sigma \in \operatorname{Sol}(\mathcal{P})} f(\sigma) \text {. }
$$

An optimal solution of $\mathcal{O}$ is a solution $\sigma$ of $\mathcal{P}$ whose objective value is optimal, i.e., $f(\sigma)=f^{*}$. We use $\operatorname{Sol}(\mathcal{O})$ to denote $\operatorname{Sol}(\mathcal{P})$ in the following.

The key idea behind this paper is that symmetries can be systematically derived through composition of CSPs (or COPs). The next definition captures compositions of CSPs formally.

Definition 4. Let $\mathcal{P}_{1}=\left\langle V, D, C_{1}\right\rangle$ and $\mathcal{P}_{2}=\left\langle V, D, C_{2}\right\rangle$ be two CSPs. The composition of $\mathcal{P}_{1}$ and $\mathcal{P}_{2}$, denoted by $\mathcal{P}_{1} \wedge \mathcal{P}_{2}$, is the CSP $\mathcal{P}=\left\langle V, D, C_{1} \wedge C_{2}\right\rangle$, whose solutions satisfy $\operatorname{Sol}(\mathcal{P})=\operatorname{Sol}\left(\mathcal{P}_{1}\right) \cap \operatorname{Sol}\left(\mathcal{P}_{2}\right)$.

\section{Value and Variable Interchangeability}

There are many applications in resource allocation and scheduling where the exact values taken by the variables are not important. What is significant is which variables take the same values or, in other terms, how the variables are clustered. Other applications exhibit weaker notions of value interchangeability, such as the concept of piecewise value interchangeability where only subsets of values are interchangeable. As shown in (Van Hentenryck et al. 2003), these symmetries can be broken efficiently during search and it is thus particularly important to discover them automatically.
Definition 5. Let $\mathcal{P}=\langle V, D, C\rangle$ be a CSP. $\mathcal{P}$ is valueinterchangeable if, for each solution $\sigma \in \operatorname{Sol}(\mathcal{P})$ and each bijection $b: D \rightarrow D$, the function $b \circ \sigma \in \operatorname{Sol}(\mathcal{P})$.

Example 6. Let $V \supseteq\left\{v_{1}, v_{2}, v_{3}\right\}$. The CSP $\mathcal{P}=$ $\left\langle V, D\right.$, allDifferent $\left.\left(v_{1}, v_{2}, v_{3}\right)\right\rangle$ is value-interchangeable.

We now define piecewise value-interchangeability.

Definition 7. Let $\mathcal{D}=\left\{D_{1}, \ldots, D_{n}\right\}$ be a partition of $D$. A bijection $b: D \rightarrow D$ is piecewise interchangeable over $\mathcal{D}$ if $\forall v \in D_{i}: b(v) \in D_{i} \quad(1 \leq i \leq n)$.

Definition 8. Let $\mathcal{P}=\langle V, D, C\rangle$ be a CSP and $\mathcal{D}$ be a partition of $D . \mathcal{P}$ is piecewise-value-interchangeable (PVI) over $\mathcal{D}$ if, for each solution $\sigma \in \operatorname{Sol}(\mathcal{P})$ and each piecewiseinterchangeable bijection $b$ over $\mathcal{D}, b \circ \sigma \in \operatorname{Sol}(\mathcal{P})$.

Note that, if $\mathcal{P}=\langle V, D, C\rangle$ is value-interchangeable, then it is piecewise-value-interchangeable over $\{D\}$. As a consequence, it is easy to compose these two forms of symmetries.

Example 9. Let $V \supseteq\left\{v_{1}, v_{2}, v_{3}\right\}, \quad D \quad \ni \quad 1$, and consider a constraint atmost $\left(o, d,\left\langle v_{1}, \ldots, v_{k}\right\rangle\right)$ which holds for an assignment $\sigma$ if there are at most $O$ occurrences of $d$ in $\left\langle\sigma\left(v_{1}\right), \ldots, \sigma\left(v_{k}\right)\right\rangle$. The CSP $\left\langle V, D, \operatorname{atmost}\left(2,1,\left\langle v_{1}, v_{2}, v_{3}\right\rangle\right)\right\rangle$ is PVI over $\{\{1\}, D\rangle$ $\{1\}\}$.

Value-interchangeability also applies to global functions, in which case the value of a function must not change under various forms of bijection.

Definition 10. A global function $f:(V \rightarrow D) \rightarrow \mathcal{N}$ is value-interchangeable if, for each assignment $\sigma: V \rightarrow D$ and each bijection $b: D \rightarrow D, f(\sigma)=f(b \circ \sigma)$.

Example 11. Let $V \supseteq\left\{v_{1}, \ldots, v_{5}\right\}$ and consider global functions of the form $n b \operatorname{Distinct}\left(v_{1}, \ldots, v_{k}\right)$ which, given an assignment $\sigma$, return the number of distinct values in $\left\langle\sigma\left(v_{1}\right), \ldots, \sigma\left(v_{k}\right)\right\rangle$. The global function $n b \operatorname{Distinct}\left(v_{1}, \ldots, v_{5}\right)$ is value-interchangeable.

Definition 12. Let $\mathcal{D}$ be a partition of $D$. A global function $f:(V \rightarrow D) \rightarrow \mathcal{N}$ is piecewise-value-interchangeable over $\mathcal{D}$ if, for each assignment $\sigma: V \rightarrow D$ and piecewiseinterchangeable bijection $b$ over $\mathcal{D}, f(\sigma)=f(b \circ \sigma)$.

These concepts can be generalized to COPs.

Definition 13. Let $\mathcal{O}=\langle V, D, C, f\rangle$ be a COP. $\mathcal{O}$ is valueinterchangeable if, for each solution $\sigma \in \operatorname{Sol}(\mathcal{O})$ and each bijection $b: D \rightarrow D, b \circ \sigma \in \operatorname{Sol}(\mathcal{O})$ and $f(\sigma)=f(b \circ \sigma)$.

Definition 14. Let $\mathcal{O}=\langle V, D, C, f\rangle$ be a COP and $\mathcal{D}$ be a partition of $D . \mathcal{O}$ is piecewise-value-interchangeable over $\mathcal{D}$ if, for each solution $\sigma \in \operatorname{Sol}(\mathcal{O})$ and each piecewiseinterchangeable bijection $b$ over $\mathcal{D}, b \circ \sigma \in \operatorname{Sol}(\mathcal{O})$ and $f(\sigma)=f(b \circ \sigma)$.

In the following, we often assume fixed sets $V$ and $D$ in examples for simplicity and talk directly about the composition and interchangeability of constraints, since they are essentially equivalent to their CSP counterparts.

It is also important to emphasize that all results presented in the next sections have direct counterparts for variable interchangeability. This is due to the fact that the definition of variable interchangeability is essentially similar to value interchangeability. Consider the simplest definition of variable interchangeability. 
Definition 15. Let $\mathcal{P}=\langle V, D, C\rangle$ be a CSP. $\mathcal{P}$ is variableinterchangeable if, for each solution $\sigma \in \operatorname{Sol}(\mathcal{P})$ and each bijection $b: V \rightarrow V$, the function $\sigma \circ b \in \operatorname{Sol}(\mathcal{P})$.

The difference is the composition order of $\sigma$ and the bijection (which also has a different signature).

\section{Composition of Constraint Symmetries}

Value symmetries arise in many applications and can be broken efficiently during search. Unfortunately, there is no general efficient algorithm for computing interchangeable values in CSPs (Freuder 1991). The key insight in this paper is that symmetries can be compositionally inferred from global constraints. More precisely, given two constraints (or CSPs) $C_{1}$ and $C_{2}$, the symmetries of their composition $C_{1} \wedge C_{2}$ can be inferred automatically from the symmetries of $C_{1}$ and $C_{2}$. The following result is immediate.

Proposition 16. Let $\mathcal{P}_{1}=\left\langle V, D, C_{1}\right\rangle$ and $\mathcal{P}_{2}=\left\langle V, D, C_{2}\right\rangle$ be two value-interchangeable CSPs. Then, their composition $\mathcal{P}_{1} \wedge \mathcal{P}_{2}$ is value-interchangeable.

The following example illustrates the result.

Example 17. Let $V \supseteq\left\{v_{1}, \ldots, v_{6}\right\}$ and let $C_{1}$ and $C_{2}$ be the constraints allDifferent $\left(v_{1}, v_{2}, v_{3}\right)$ and allDifferent $\left(v_{4}, v_{5}, v_{6}\right)$. Then $C_{1} \wedge C_{2}$ is valueinterchangeable.

Note that constraints in practice only "constrain" a subset of the variables, although they are formally defined over all variables. The next result specifies how to compose piecewise-value-interchangeable CSPs.

Proposition 18. Let $\mathcal{P}_{1}=\left\langle V, D, C_{1}\right\rangle$ and $\mathcal{P}_{2}=$ $\left\langle V, D, C_{2}\right\rangle$ be two CSPs. Assume that $\mathcal{P}_{i}$ is piecewise-valueinterchangeable over partition $\mathcal{D}_{i}$ of $D(1 \leq i \leq 2)$. Then the composition $\mathcal{P}_{1} \wedge \mathcal{P}_{2}$ is piecewise-value-interchangeable over

$\mathcal{D}=\left\{D_{1} \cap D_{2} \mid D_{1} \in \mathcal{D}_{1} \& D_{2} \in \mathcal{D}_{2} \& D_{1} \cap D_{2} \neq \emptyset\right\}$.

Proof. First observe that $\mathcal{D}$ is a partition of $D$. Now let $b$ be a piecewise-interchangeable bijection over $\mathcal{D}$. We show that $b$ is piecewise-interchangeable over $\mathcal{D}_{1}$. Indeed, consider a set $D_{1} \in \mathcal{D}_{1}$ and a value $d \in D_{1}$. By definition of $\mathcal{D}$, there exists $D_{2} \in \mathcal{D}_{2}$ such that $I=D_{1} \cap D_{2}$ and $d \in I$. Since $b$ is piecewise-interchangeable over $\mathcal{D}, b(d) \in I \subseteq D_{1}$ and $b$ is piecewise-interchangeable over $\mathcal{D}_{1}$. Similarly, we can show that $b$ is piecewise-interchangeable over $\mathcal{D}_{2}$. As a consequence, if $\sigma \in \operatorname{Sol}\left(\mathcal{P}_{1} \wedge \mathcal{P}_{2}\right)$, then $b \circ \sigma \in \operatorname{Sol}\left(\mathcal{P}_{1}\right)$ and $b \circ \sigma \in \operatorname{Sol}\left(\mathcal{P}_{2}\right)$. Hence, $b \circ \sigma \in \operatorname{Sol}\left(\mathcal{P}_{1} \wedge \mathcal{P}_{2}\right)$.

Example 19. Let $D=\{1, \ldots, 10\}$ and let $C_{1}$ and $C_{2}$ be the constraints atmost $\left(1,1,\left\langle v_{1}, \ldots, v_{5}\right\rangle\right)$ and $\operatorname{atmost}\left(2,2,\left\langle v_{1}, \ldots, v_{5}\right\rangle\right)$ which are PVI over $\mathcal{D}_{1}=$ $\{\{1\},\{2, \ldots, 10\}\}$ and $\mathcal{D}_{2}=\{\{2\},\{1,3, \ldots, 10\}\}$ respectively. The composition $C_{1} \wedge C_{2}$ is PVI over

$$
\mathcal{D}=\{\{1\},\{2\},\{3, \ldots, 10\}\} .
$$

It is important to emphasize that the derivation of symmetries using propositions 16 and 18 is polynomial in $|D|$. As a consequence, the compositional symmetry analysis of a CSP is polynomial in $|D|$ and the number of constraints. Of course, it is not guaranteed to be precise, i.e., it may not report all symmetries in the application. However, whenever global constraints are used to model an application, the symmetries appear naturally and the loss of precision is often avoided. Futhermore, we discuss this later in the paper how reformulations may help in addressing this issue.

\section{Composition of Function Symmetries}

This section shows how to compose function symmetries from global functions. It also shows how to infer symmetries in COPs and how function symmetries can be used to infer symmetries on numerical constraints.

Proposition 20. Let $f_{1}$ and $f_{2}$ be two global functions of signature $(V \rightarrow D) \rightarrow \mathcal{N}$. If $f_{1}$ and $f_{2}$ are valueinterchangeable, then so are $f_{1} \star f_{2}(\star \in\{+,-, \times\})$.

Of course, the result can be generalized to other operators.

Example 21. Let $V \supseteq\left\{v_{1}, \ldots, v_{6}\right\}$ and let $f_{1}$ and $f_{2}$ be the global functions nbDistinct $\left(v_{1}, v_{2}, v_{3}\right)$ and $n b \operatorname{Distinct}\left(v_{4}, v_{5}, v_{6}\right)$. Then, the global function $3 f_{1}+4 f_{2}$ is value-interchangeable.

Proposition 22. Let $f_{1}:(V \rightarrow D) \rightarrow \mathcal{N}$ and $f_{2}:$ $(V \rightarrow D) \rightarrow \mathcal{N}$ be two global functions. If $f_{1}$ and $f_{2}$ are piecewise-value-interchangeable over $\mathcal{D}_{1}$ and $\mathcal{D}_{2}$ respectively, then $f_{1} \star f_{2}$, where $\star \in\{+,-, \times\}$, is piecewisevalue-interchangeable over

$\mathcal{D}=\left\{D_{1} \cap D_{2} \mid D_{1} \in \mathcal{D}_{1} \& D_{2} \in \mathcal{D}_{2} \& D_{1} \cap D_{2} \neq \emptyset\right\}$.

We now show how to derive symmetries for COPs by considering both the constraint and the objective function.

Proposition 23. Let $\mathcal{O}=\langle V, D, C, f\rangle$ be a COP and $\mathcal{P}=\langle V, D, C\rangle$. If $\mathcal{P}$ and $f$ are value-interchangeable, then $\mathcal{O}$ is value-interchangeable. If $\mathcal{P}$ is piecewise-valueinterchangeable over partition $\mathcal{D}_{1}$ of $D$ and $f$ is piecewisevalue-interchangeable over partition $\mathcal{D}_{2}$ of $D$, then $\mathcal{O}$ is piecewise-value-interchangeable over

$\mathcal{D}=\left\{D_{1} \cap D_{2} \mid D_{1} \in \mathcal{D}_{1} \& D_{2} \in \mathcal{D}_{2} \& D_{1} \cap D_{2} \neq \emptyset\right\}$.

In many applications, constraints are built from global functions and arithmetic operators. The next proposition shows how to derive symmetries for such constraints.

Proposition 24. Let $f:(V \rightarrow D) \rightarrow \mathcal{N}$ be a global function and $\mathcal{D}$ be a partition of $D$. If $f$ is piecewisevalue-interchangeable over $\mathcal{D}$, then the $\operatorname{CSP}\langle V, D, f \approx 0\rangle$ is piecewise-value-interchangeable over $\mathcal{D}$ as well, where $\approx \in\{>, \geq,=, \neq, \leq,<\}$.

\section{Scene Allocation}

We now illustrate how these results can be used to detect value symmetries on the scene-allocation problem, which consists of producing a movie at minimal cost by deciding when to shoot scenes. Each scene involves a number of actors and at most 5 scenes a day can be filmed. All actors of a scene must be present on the day the scene is shot. The actors have fees representing the amount to be paid per day they spend in the studio. The goal of the application is to 


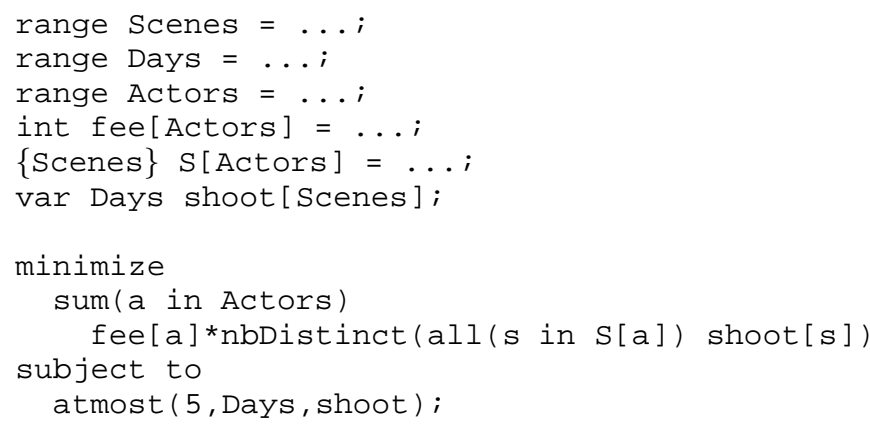

Figure 1: The Scene Allocation Model

minimize the production costs and an optimal solution is an assignment of scenes to days which minimizes the production costs. On some reasonably small instances, a state-ofthe-art MIP solver took about 2 minutes and a CP solver took about 8 minutes for solving the problem. By removing value symmetries during search, the execution time of the CP solver fell to below 10 seconds (Van Hentenryck 2002).

It is also interesting to quote (Van Hentenryck et al. 2003) here: "It should be apparent that the exact days assigned to the scenes have no importance in this application and are fully interchangeable. What is important is how the scenes are clustered together. Our approach does not aim at discovering this fact; rather it focuses on how to exploit it to eliminate the symmetries it induces." The main contribution of this paper is entirely orthogonal: it shows how the value interchangeability of the scene allocation problem can be automatically derived from the properties of the constraints.

Consider Figure 1 which depicts an OPL-like model for scene allocation, where the instance data is given in a separate file as typical. The first three lines specify the various ranges for scenes, days, and actors. The next two lines specify the fee of each actor and the set of scenes $S$ [a] which actor a plays in. The next line specifies the variables and shoot [s] represents the day assigned to scene $s$. The constraint atmost (5, Days, shoot) is a global cardinality constraint which specifies that at most 5 scenes can be shot every day. The objective function sums the fees of each actor, each actor being paid her fee for each different day in which one of her scenes is shot. Indeed, the expression all (s in $\mathrm{S}[\mathrm{a}]$ ) shoot [s] collects the variables associated with the scenes of actor a in an array of variables, which is used in the function nbDist inct. Observe now that constraint atmost ( 5 , Days, shoot) is valueinterchangeable. The global function nbDistinct is also value-interchangeable (see Example 11). By Proposition 20, the objective function is value-interchangeable. Hence, by Proposition 23, the scene-allocation model in Figure 1 is value-interchangeable. In summary, as mentioned earlier, once the value symmetries of the global objects are known, it is possible to derive value symmetries of the entire model using the results of this paper.

It is also useful to stress the benefits of global constraints. The value symmetries derived on the model above are dramatically more complicated to detect on the MIP model. Indeed, the values are not even explicit in that model, which encodes the scene assignment in terms of 0/1 variables.

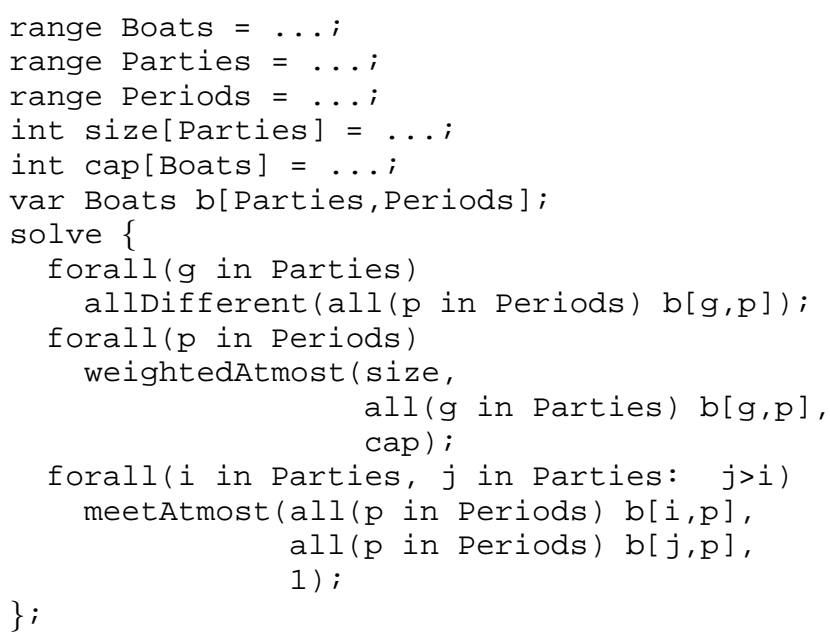

Figure 2: The Progressive Party Model

\section{Progressive Party Problem}

The progressive party problem is a traditional benchmark which is often used to compare constraint programming, mathematical programming, and local search. Figure 2 depicts an OPL-like model for this problem, which is a direct translation of the Comet model in (Michel \& Van Hentenryck 2002). The first three lines specify the ranges, i.e., the boats, the parties, and the periods. The next two lines specify the size of the parties and the capacities of the boats. The variables are declared next and assign a boat $b[g, p]$ to party $g$ at period $p$. The first set of constraints specifies that a party never visits the same boat twice. The second set of constraints are weighted cardinality constraints which specify that the sizes of the parties visiting a boat during a period cannot exceed the boat capacity. The final set of constraints are again cardinality constraints specifying that two parties meet at most once: a constraint meetAtmost $\left(\left\langle v_{1}, \ldots, v_{p}\right\rangle,\left\langle w_{1}, \ldots, w_{p}\right\rangle, k\right)$ holds for an assignment $\sigma$ if $\#\left\{i \in 1 . . p \quad \sigma\left(v_{i}\right)=\sigma\left(w_{i}\right)\right\} \leq$ $k$. Observe that the allDifferent constraints are valueinterchangeable. The meetAtmost constraints are also valueinterchangeable. The interesting part in this model are the weightedAtmost constraints. A constraint

weightedAtmost $\left(\left\langle s_{1}, \ldots, s_{n}\right\rangle,\left\langle v_{1}, \ldots, v_{n}\right\rangle,\left\langle c_{1}, \ldots, c_{m}\right\rangle\right)$

holds for an assignment $\sigma$ if $\forall k \in 1 . . m: \sum_{i \in S_{k}} s_{i} \leq c_{k}$ where $S_{k}=\left\{i \in 1 . . n \mid \sigma\left(v_{i}\right)=k\right\}$. This constraint is piecewise-value-interchangeable over $\mathcal{D}=\left\{D_{1}, \ldots, D_{m}\right\}$, where $D_{k}=\left\{i \in 1 . . n \mid c_{i}=c_{k}\right\}$. As a consequence, our compositional derivation automatically infers that boats with the same capacity are piecewise-valueinterchangeable. Note that a similar derivation for the variables infers that parties with the same sizes are piecewisevariable-interchangeable.

\section{Reformulations}

The symmetry derivations presented earlier can often be strenghtened by model reformulations which can be seen as adaptations to constraint satisfaction of "presolve" techniques used in mixed-integer programming (Johnson, 
Nemhauser, \& Savelsbergh 2000). For space reasons, we only present two reformulations, aggregation and projection.

Aggregation The symmetry derivations presented earlier may be suboptimal as the following example indicates.

Example 25. Let $V \supseteq\left\{v_{1}, \ldots, v_{3}\right\}$ and $D \supseteq\{1,2\}$. Constraint $C_{1}=\operatorname{atmost}\left(2,1,\left\langle v_{1}, v_{2}, v_{3}\right\rangle\right)$ is PVI over $\mathcal{D}=$ $\{\{1\}, D \backslash\{1\}\}$. Constraint $C_{2}=\operatorname{atmost}\left(2,2,\left\langle v_{1}, v_{2}, v_{3}\right\rangle\right)$ is PVI over $\mathcal{D}=\{\{2\}, D \backslash\{2\}\}$. By Proposition 18, $C_{1} \wedge C_{2}$ is PVI over $\mathcal{D}=\{\{1\},\{2\}, D \backslash\{1,2\}\}$. However, $C_{1} \wedge C_{2}$ is also PVI over $\mathcal{D}=\{\{1,2\}, D \backslash\{1,2\}\}$, which is stronger.

This precision loss can be remedied by modeling the problem more globally using, say, a global cardinality constraint (Régin 1999). Again, the observation is that global constraints are fundamental tools to derive stronger symmetries.

Example 26. Consider a global cardinality constraint $\operatorname{atmost}\left(\left\langle o_{1}, \ldots, o_{k}\right\rangle,\left\langle d_{1}, \ldots, d_{k}\right\rangle,\left\langle v_{1}, \ldots, v_{n}\right\rangle\right) \quad$ which holds for an assignment $\sigma$ if there exist at most $o_{i}$ occurrences of $d_{i}$ in $\left\langle\sigma\left(v_{1}\right), \ldots, \sigma\left(v_{n}\right)\right\rangle(1 \leq i \leq k)$. It is PVI over $\left\{D_{1}, \ldots, D_{k}, D \backslash\left(D_{1} \cup \cdots \cup D_{k}\right)\right\}$ where $D_{i}=\left\{d_{j} \mid o_{j}=o_{i} \& 1 \leq j \leq k\right\} \quad(1 \leq i \leq k)$. For instance, $\operatorname{atmost}\left(\langle 1,2,1\rangle,\langle 1,2, \overline{3}\rangle,\left\langle v_{1}, \ldots, v_{n}\right\rangle\right)$ is PVI over $\{\{1,3\},\{2\}, D \backslash\{1,2,3\}\}$ since $D_{1}=D_{3}=\{1,3\}$.

As a consequence, automated tools for symmetry detection should provide aggregation operators exploiting the semantics of constraints. They can be specified as follows.

Definition 27. Let $C_{1}$ and $C_{2}$ be two constraints of signature $\mathcal{C}=(V \rightarrow D) \rightarrow$ Bool. A compositional aggregator is a binary operator $\otimes$ of signature $(\mathcal{C} \times \mathcal{C}) \rightarrow \mathcal{C}$ such that $C_{1} \otimes C_{2}$ is a single constraint equivalent to $C_{1} \wedge C_{2}$.

Example 28. Let $V \supseteq\left\{v_{1}, v_{2}, v_{3}\right\}, D \supseteq\{1,2\}$ and constraints $C_{1}=\operatorname{atmost}\left(2,1,\left\langle v_{1}, v_{2}, v_{3}\right\rangle\right)$ and $C_{2}=\operatorname{atmost}\left(2,2,\left\langle v_{1}, v_{2}, v_{3}\right\rangle\right) . \quad \mathrm{A}$ compositional aggregator of $C_{1}$ and $C_{2}$ may return the constraint $\operatorname{atmost}\left(\langle 2,2\rangle,\langle 1,2\rangle,\left\langle v_{1}, \ldots, v_{3}\right\rangle\right)$.

Projection Projections, the second class of reformulations considered in this paper, are important in many applications. On the one hand, they are often useful when a general model (e.g., a round-robin sport-scheduling model) is specialized to a specific problem (e.g., the ACC basketball schedule for the 2004 season) by introducing, among others, some fixed decisions. On the other hand, they are useful in deriving dynamic symmetries, i.e., symmetries not present in the original problem but arising after a number of variable assignments. The following example illustrates the significance of projections when deriving symmetries.

Example 29. Let $V=\left\{v_{1}, \ldots, v_{5}\right\}$, let $C_{1}$ be the constraint atmost $\left(\langle 3,2\rangle,\langle 1,2\rangle,\left\langle v_{1}, \ldots, v_{5}\right\rangle\right)$ and $C_{2}$ be $v_{1}=1$. The $\operatorname{CSP}\left\langle V, D, C_{1} \wedge C_{2}\right\rangle$ is derived to be PVI over $\mathcal{D}=$ $\{\{1\},\{2\}, D \backslash\{1,2\}\}$ since $C_{1}$ is PVI over $\{\{1\},\{2\}, D \backslash$ $\{1,2\}\}$ and $C_{2}$ is PVI over $\{\{1\}, D \backslash\{1\}\}$, which is as strong as possible. However, consider $V^{\prime}=V \backslash\left\{v_{1}\right\}$ and the constraint $C$ defined as $\operatorname{atmost}\left(\langle 2,2\rangle,\langle 1,2\rangle,\left\langle v_{2}, \ldots, v_{5}\right\rangle\right)$. The CSP $\left\langle V^{\prime}, D, C\right\rangle$ is PVI over $\mathcal{D}=\{\{1,2\}, D \backslash\{1,2\}\}$.

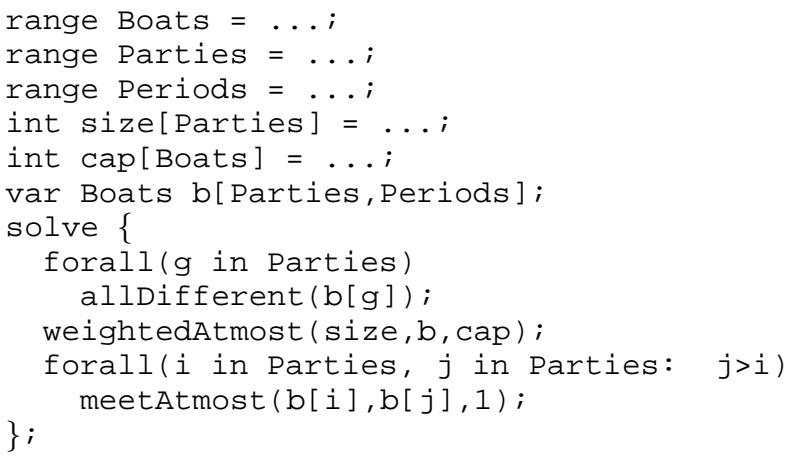

Figure 3: The Progressive Party Matrix Model

This example indicates that more symmetries may be available on subproblems when some variables are projected out. Moreover, since the assignment of values to variables is the fundamental operation of many search procedures, projections are an important tool to derive symmetries dynamically. As a consequence, symmetry detection tools should ideally include projection operators exploiting the semantics of primitive constraints.

Definition 30. Let $C$ be a constraint of signature $\mathcal{C}=(V \rightarrow$ $D) \rightarrow$ Bool, and $V^{\prime}=V \backslash\{v\}$. A projection operator for $\mathcal{C}$ wrt $v=d$ is a function $\uparrow_{v=d}$ of signature $\mathcal{C} \rightarrow \mathcal{C}^{\prime}$, where $\mathcal{C}^{\prime}=\left(V^{\prime} \rightarrow D\right) \rightarrow$ Bool, satisfying

$$
\operatorname{Sol}(\langle V, D, C \wedge v=d\rangle)=\operatorname{Sol}\left(\left\langle V, D, C \uparrow_{v=d} \wedge v=d\right\rangle .\right.
$$

The key intuition here is that constraint $C \uparrow_{v=d}$ is only expressed in terms of variables in $V^{\prime}$ and does not add or remove any solution to the original problem.

\section{Symmetries in Matrix Models}

This section considers the derivation of variable symmetries in matrix models, which have been found useful in a variety of applications involving symmetries. In particular, we show how the techniques presented earlier apply to the detection of column symmetries in matrix models. (The derivation of row symmetries is similar.) Figure 3 presents a specification of the progressive party problem using matrix modeling. It is essentially similar to the model presented earlier but uses matrices and rows of matrices directly in constraints. We now show how to systematically derive columninterchangeability on this model.

Formally, a matrix $M$ of variables can be modelled as a bijection $X \times Y \rightarrow V$, where $X$ are the row indices of $M$, $Y$ its column indices, and $V$ its set of variables. For clarity, we use traditional notations: $M[i, j]$ denotes the variable in row $i$ and in column $j, M[i]$ row $i$, and $M[*, j]$ column $j$. We assume that all matrices are defined over row indices $X$ and column indices $Y$.

Definition 31. A matrix-CSP (MCSP) is a triple $\langle M, D, C\rangle$, where $M$ is a matrix of variables, $D$ denotes the set of values for these variables, and $C:(M \rightarrow D) \rightarrow$ Bool specifies which assignments of values to the variables are solutions. A solution to an MCSP $\mathcal{P}=\langle M, D, C\rangle$ is a function $\sigma$ : $M \rightarrow D$ such that $C(\sigma)=$ true. The set of solutions to $\mathcal{P}$ is denoted by $\operatorname{Sol}(\mathcal{P})$. 
The next definitions specify column interchangeability, a "global" form of variable interchangeability.

Definition 32. A column permutation for a matrix $M$ is a function $\rho: M \rightarrow M$ such that

$$
M[i, j]=\rho(M)[i, b(j)] \quad(i \in X \& j \in Y)
$$

for some bijection $b: Y \rightarrow Y$.

Definition 33. An MCSP $\mathcal{P}=\langle M, D, C\rangle$ is columninterchangeable if, for each solution $\sigma \in \operatorname{Sol}(\mathcal{P})$ and each column permutation $\rho: M \rightarrow M$, the function $\sigma \circ \rho \in$ $\operatorname{Sol}(\mathcal{P})$.

Proposition 34. Let $\mathcal{P}_{1}=\left\langle M, D, C_{1}\right\rangle$ and $\mathcal{P}_{2}=$ $\left\langle M, D, C_{2}\right\rangle$ be two column-interchangeable MCSPs. Then, their composition $\mathcal{P}_{1} \wedge \mathcal{P}_{2}$ is column-interchangeable.

Consider the matrix model in Figure 3. Constraints allDifferent and meetAtmost are column-interchangeable. Indeed, the variable (resp. pair) order is not significant in allDifferent (resp. meetAtmost) and both are applied on rows of the matrix. The global weightedAtmost constraint is columninterchangeable, since it applies the same constraint to all columns. It is an aggregation of

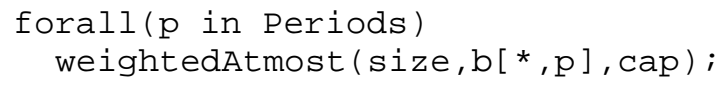

which cannot be shown column-interchangeable compositionally. We conclude this section by generalizing the results to piecewise interchangeability.

Definition 35. Let $\mathcal{Y}$ be a partition over $Y$. A piecewise column permutation over $\mathcal{Y}$ for a matrix $M$ is a function $\rho: M \rightarrow M$ such that

$$
M[i, j]=\rho(M)[i, b(j)] \quad(i \in X \& j \in Y)
$$

for some piecewise-interchangeable bijection $b$ over $\mathcal{Y}$.

Definition 36. Let $\mathcal{Y}$ be a partition over $Y$. An $\operatorname{MCSP} \mathcal{P}=$ $\langle M, D, C\rangle$ is piecewise-column-interchangeable over $\mathcal{Y}$ if, for each solution $\sigma \in \operatorname{Sol}(\mathcal{P})$ and each piecewise column permutation $\rho$ over $\mathcal{Y}$, the function $\sigma \circ \rho \in \operatorname{Sol}(\mathcal{P})$.

Proposition 37. Let $\mathcal{P}_{1}=\left\langle M, D, C_{1}\right\rangle$ and $\mathcal{P}_{2}=$ $\left\langle M, D, C_{2}\right\rangle$ be two piecewise-column-interchangeable $M C$ SPs over $\mathcal{Y}_{1}$ and $\mathcal{Y}_{2}$ respectively. Then, their composition $\mathcal{P}_{1} \wedge \mathcal{P}_{2}$ is piecewise-column-interchangeable over

$$
\mathcal{Y}=\left\{Y_{1} \cap Y_{2} \mid Y_{1} \in \mathcal{Y}_{1} \& Y_{2} \in \mathcal{Y}_{2} \& Y_{1} \cap Y_{2} \neq \emptyset\right\} .
$$

These results naturally generalize to matrix-COPS.

\section{Conclusion}

This paper reconsidered the problem of discovering symmetries in constraint satisfaction problems by exploiting one of the fundamental aspects of constraint programming: the ability of global constraints to capture combinatorial substructures. The paper showed that, once the symmetries of global constraints are specified, various classes of symmetries can be derived precisely and efficiently in a compositional fashion. The paper studied value and variable interchangeability, as well as column and row interchangeability in matrix models. It also stressed the benefits of traditional reformulations such as aggregation and projection to strengthen symmetry detection. The potential of this novel approach was demonstrated on two non-trivial applications.

It is interesting to relate this research to the automatic modeling project of (Bakewell, Frisch, \& Miguel 2003), which uses compositional refinement to transform abstract specifications into constraint programs. Since these transformations may introduce symmetries, (Bakewell, Frisch, \& Miguel 2003) propose to annotate the refinement rules with the symmetries so that they can be broken subsequently. Our bottom-up derivation approach is entirely orthogonal to their top-down refinement approach: It could in fact be applied as a first step to deduce properties of models before refinement. Both works also address the need for more automation for non-experts, a feature which is currently lacking in constraint programming when compared to MIP technology.

\section{References}

Backofen, R., and Will, S. 1999. Excluding Symmetries in Constraint-Based Search. In $C P^{\prime} 99$.

Bakewell, A.; Frisch, A.M.; and Miguel, I. 2003. Towards Automatic Modelling of Constraint Satisfaction Problems: A System Based on Compositional Refinement. In CP'03 Workshop on Modelling and Reformulating Constraint Satisfaction Problems.

Barnier, N., and Brisset, P. 2002. Solving the Kirkman's schoolgirl problem in a few seconds. In $C P^{\prime} 02$.

Beldiceanu, N., and Contejean, E. 1994. Introducing Global Constraints in CHIP. Journal of Mathematical and Computer Modelling 20(12):97-123.

Fahle, T.; Schamberger, S.; and Sellmann, M. 2001. Symmetry breaking. In $C P^{\prime} 01$.

Flener, P.; Frisch, A. M.; Hnich, B.; Kiziltan, Z.; Miguel, I.; Pearson, J.; and Walsh, T. 2002. Breaking row and column symmetries in matrix models. In $\mathrm{CP}^{\prime} \mathrm{O2}$.

Focacci, F., and Milano, M. 2001. Global cut framework for removing symmetries. In $C P^{\prime} 01$.

Focacci, F.; Lodi, A.; and Milano, M. 2002. OptimizationOriented Global Constraints. Constraints 7(3-4):351-365.

Freuder, E. 1991. Eliminating interchangeable values in constraint satisfaction problems. In $A A A I^{\prime} 91$.

Gent, I. P., and Smith, B. 2000. Symmetry breaking during search in constraint programming. In ECAI-2000.

Johnson, E.; Nemhauser, G.; and Savelsbergh, M. 2000. Progress in Linear Programming-Based Algorithms for Integer Programming: An Exposition. INFORMS Journal on Computing 12:2-23.

Michel, L., and Van Hentenryck, P. 2002. A constraint-based architecture for local search. In OOPSLA'02.

Puget, J.-F. 2002. Symmetry Breaking Revisited. In $C P^{\prime} O 2$.

Puget, J.-F. 2003. Symmetry breaking using stabilizers. In $C P^{\prime} 03$.

$\mathrm{R}^{\prime}$ egin, J.-C. 1994. A filtering algorithm for constraints of difference in CSPs. In AAAI-94.

R'egin, J.-C. 1999. Arc Consistency for Global Cardinality Constraints with Costs. In $C P^{\prime} 99$.

Van Hentenryck, P.; Flener, P.; Pearson, J.; and Ågren, M. 2003. Tractable symmetry breaking for CSPs with interchangeable values. In IJCAI'03.

Van Hentenryck, P. 2002. Constraint and Integer Programming in OPL. Informs Journal on Computing 14(4):345-372. 